\title{
Searches for long lived particles at the Tevatron
}

\author{
Tulika Bose \\ Department of Physics, Brown University \\ 182 Hope Street, Providence, RI 02912, U.S.A.
}

\begin{abstract}
Several searches have been performed for long lived particles using data collected by the CDF and D $\varnothing$ detectors at the Fermilab Tevatron collider. These include searches for charged massive stable particles, stopped gluinos, neutral long-lived particles decaying to muons, and magnetic monopoles. These proceedings [1] review recent experimental results from Run II analyses.
\end{abstract}

\section{Introduction}

Exotic long lived particles are predicted by several extensions of the standard model. GaugeMediated Supersymmetry Breaking (GMSB) models [2] usually postulate a light gravitino as the lightest supersymmetric particle (LSP). In such models all supersymmetric particles decay via cascades to the next-to-lightest supersymmetric particle or NLSP. The decay of the NLSP itself is determined by its coupling to gravity. Large coupling strengths correspond to a small symmetry breaking scale and result in a short lifetime for the NLSP while small coupling strengths are associated with a large symmetry breaking scale and consequently a long lifetime for the NLSP. Neutralinos and stau leptons are examples of such long-lived NLSP candidates. [3].

In Anomaly Mediated Supersymmetric Breaking (AMSB) models [4] long lifetimes arise from small mass splittings between the LSP and the NLSP. If the mass difference is small enough, phase space suppression impedes the decay of the NLSP resulting in long-lived NLSP candidates like the chargino.

In Split supersymmetry models [5], gluino decays are suppressed owing to the large masses of the SUSY scalars, and consequently the gluino can be long-lived. Long lived particles are also predicted by other exotic models like universal extra dimensions, models with R-parity violation, and hidden valley models [6].

In most of the above cases, long lived particles have very distinct experimental signatures. They are often considered stable and non-interacting since they live long enough to escape the detector before decaying. In some cases, such particles may interact with the detector or decay into particles which interact. These would then register as delayed hits in the detector. Many of these long-lived particles are also quite massive $(>50 \mathrm{GeV})$ and have small values of $\beta$ or $v / c$. Some are also charged and thereby can be associated with large amounts of energy deposition. Some typical examples of such particles are stop squarks and stau leptons which interact in the detector like heavy muons with no strong interactions or electromagnetic showers.

\section{Tevatron searches}

\subsection{Charged Massive Stable Particles}

Charged massive stable particles would register in the CDF and DØ detectors as slowlymoving, highly ionizing and highly penetrating particles with large transverse momentum

DIS 2008 
and typically reconstructed as muon candidates. CDF has searched for CHArged Massive Stable ParticleS (CHAMPS) which would register as delayed hits in the Time of Flight (TOF) detector and the Central Tracker (COT) [7]. To identify such particles a data sample is collected using trigger information from the muon chambers and the velocity and momentum of the candidate tracks are used to calculate the time-of-flight mass of the candidate particle. The track velocity itself is measured by dividing the path length of the track by its time-of-flight which is obtained using the TOF detector. Figure 1 (left) shows the observed and predicted TOF mass distributions for signal region muons. Since the data is consistent with the predicted background, CDF sets model-independent upper limits on the production cross section of both strongly interacting and weakly interacting CHAMPs. Figure 1 (right) shows the observed 95\% C.L. limit on the production cross section for stable stop squarks using $\approx 1.03 \mathrm{fb}^{-1}$ of data. The intersection of this curve with the NLO theoretical cross section can be used to set a 95\% C.L. upper limit on the mass of a stable stop at $250 \mathrm{GeV} / \mathrm{c}^{2}$.
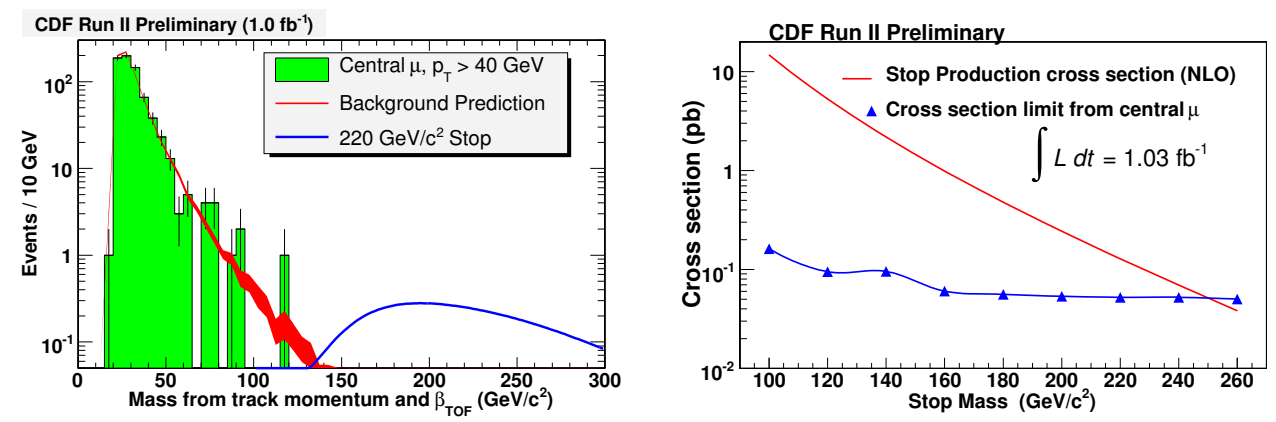

Figure 1: Observed (histogram) and predicted (curve) time-of-flight mass distributions for candidate tracks in the muon sample (right plot). The blue line for large masses denotes the additional events expected for a $220 \mathrm{GeV}$ stop signal. The plot on the right shows the theoretical NLO cross section for stop production, plotted as a function of stop mass (red), along with the $95 \%$ C.L. cross section limit.

DØ has searched for Charged Stable Massive Particles or CMSPs using $\sim 390 \mathrm{pb}^{-1}$ of data [8]. Timing information from the muon detectors was used to search for pair-produced CMSPs like stau leptons and charginos. The data was found to be consistent with the background prediction and $95 \%$ C.L.limits were set on the production cross section. Using the NLO theoretical cross section numbers from Prospino2 [9], DØ excluded higgsino-like charginos with mass below $140 \mathrm{GeV}$ and gaugino-like charginos with mass less than $174 \mathrm{GeV}$.

\subsection{Stopped Gluinos}

Long-lived gluinos are postulated by split supersymmetry and other new models like Gaugemediated SUSY. These gluinos can hadronize into $R$-hadrons which are essentially colorless bound states of a gluino and other quarks or gluons. Approximately 20-30\% of R-hadrons at the Tevatron can become charged through nuclear interactions, lose all of their momentum through ionization, and come to rest in the surrounding material [10]. DØ has performed a direct search for such stopped gluinos using a data sample corresponding to $\sim 410 \mathrm{pb}^{-1}$ of 
integrated luminosity [11].

The analysis looks for stopped gluinos which decay into a gluon and a neutralino $(\tilde{g} \rightarrow$ $\left.g \bar{\chi}_{1}^{0}\right)$. The typical signature consists largely of an empty event with a single large transverse energy deposit in the calorimeter, reconstructed as a jet, and large missing transverse energy. No evidence for a signal was found and upper limits were placed on the stopped gluino cross section as a function of the gluino mass for three different assumptions of neutralino mass (see Fig. 2). Also shown in the figure are the predicted cross sections for stopped gluinos which include both the production rate and as well as its probability to stop. Figure 2 (left) assumes a small gluino lifetime ( $<3$ hours) while Fig. 2 (right) shows the $95 \%$ C.L. upper limits for different assumptions of gluino lifetime with the limits becoming weaker for very large lifetimes owing to the fact that signal events are not recorded between accelerator stores.
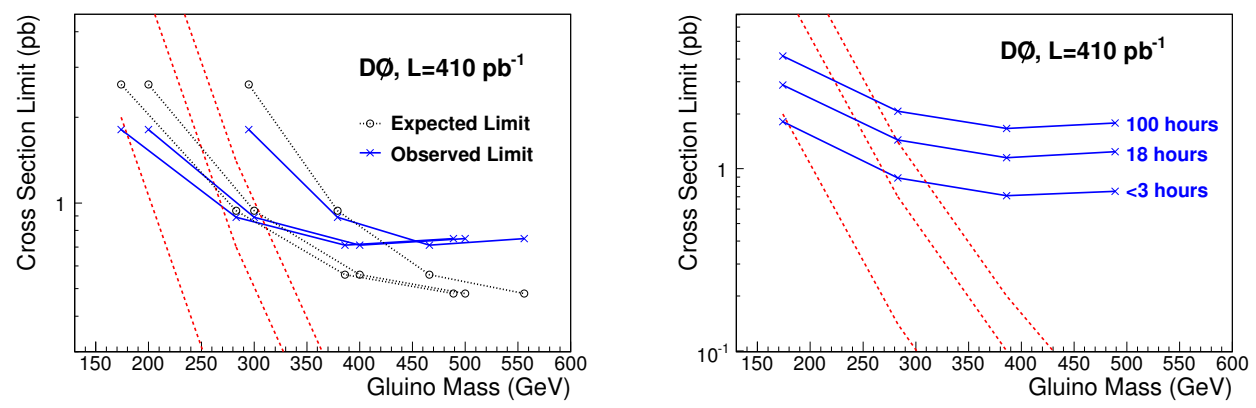

Figure 2: Left: The expected and observed upper limits on the cross section of stopped gluinos for three choices of the $\bar{\chi}_{1}^{0}$ mass $(50,90$ and $200 \mathrm{GeV}$, from left to right). A $100 \%$ branching rate of $\tilde{g} \rightarrow g \bar{\chi}_{1}^{0}$ and a small gluino lifetime are assumed. Right: Upper limits on the cross-section of stopped gluinos for various assumptions of the gluino lifetime, for a $\bar{\chi}_{1}^{0}$ mass of $50 \mathrm{GeV}$. Also shown on the plots (dashed red lines) are the theoretical stopped gluino cross sections for varying assumptions of the neutral to charged R-hadron conversion cross section $(0.3,3$, and $30 \mathrm{mb})$.

The above results exclude a cross section of $\sim 1 \mathrm{pb}$ for gluinos stopping in the $\mathrm{D} \varnothing$ calorimeter and later decaying to a neutralino and a gluon. For a neutralino mass of $50 \mathrm{GeV}$, stopped gluinos with mass less than $270 \mathrm{GeV}$ can be excluded, assuming a gluino lifetime of less than 3 hours, a $100 \%$ branching fraction for , and a neutral to charged R-hadron conversion cross section of $3 \mathrm{mb}$.

\subsection{Neutral long lived particles}

D $\varnothing$ has searched for light, neutral, long-lived particles $\left(N_{L L}^{0}\right)$ that are pair-produced in $p \bar{p}$ collisions using $380 \mathrm{pb}^{-1}$ of integrated luminosity [12]. The search was inspired by an excess of di-muon events observed by the $\mathrm{NuTeV}$ experiment [13]. The final state studied by DØ involved the decay of an $N_{L L}^{0}$ into two muons and possibly a neutrino after the $N_{L L}^{0}$ had traveled at least $5 \mathrm{~cm}$. Since no signal was found a limit was set. Fig. 3 shows the $95 \%$ (99\%) C.L. limit on the cross section $\sigma\left(p \bar{p} \rightarrow N_{L L}^{0} N_{L L}^{0} X\right.$ times the branching fraction $\left(N_{L L}^{0} \mu^{+} \mu^{-}+X\right)$ as a function of lifetime. The $95 \%$ C.L. limit for a mass of 
$10 \mathrm{GeV}$ and lifetime of $4 \times 10^{-11} \mathrm{~s}$ was found to be $0.14 \mathrm{pb}$. This result was also used to exclude an interpretation of the $\mathrm{NuTeV}$ excess of di-muon events in a large class of models.

\subsection{Magnetic monopoles}

Magnetic monopoles were first hypothesized by Dirac in 1931 [14]. He predicted that these monopoles would be accelerated by a magnetic field similar to the case where electrons are accelerated in an electric field. These monopoles would be heavy and highly ionizing. CDF has searched for such monopoles by triggering on large light pulses in the time-of-flight scintillator bars [15]. In the tracking detector, monopoles would lead to highly ionizing hits in a pattern resembling a high $p_{T}$ track. Since no monopole candidates were found in $\sim 36 \mathrm{pb}^{-1}$ of data, an upper limit was set on the cross-section. This limit excludes monopole production for cross sections greater than $0.2 \mathrm{pb}$ at the $95 \%$ C.L. for monopole masses between 200 and $700 \mathrm{GeV}$.

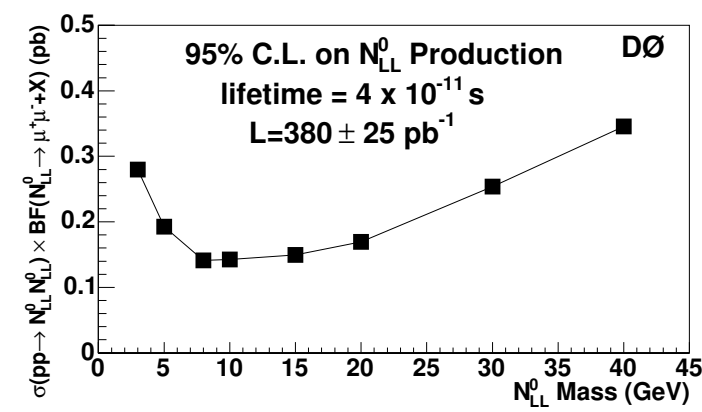

Figure 3: The limit on the pair production cross section times branching ratio for a neutral, weakly interacting particle that decays to two muons and an unobserved particle as a function of particle mass for a lifetime of $4 \times 10^{-11}$ seconds.

\section{Conclusions}

There is an exciting hunt ongoing at the Tevatron for long-lived particles. These searches cover a rich variety of exotic particles ranging from stop squarks, charginos, and neutralinos to magnetic monopoles. There is additional data already accumulated and many novel techniques are being developed to explore new possibilities. Stay tuned!

\section{References}

[1] Slides:http://indico . cern. ch/contributionDisplay $\cdot$ py? contribId=78\&sessionId=15\&conf Id=24657

[2] M. Dine, A.E. Nelson, Y. Nir, and Y. Shirman, Phys. Rev. D 53, 2658 (1996).

[3] J. Feng and T. Moroi, Phys. Rev. D 58, 035001 (1998); J. Feng, S. Su, and F. Takayama, Phys. Rev. D 70, 063514 (2004).

[4] J. Gunion and S. Mrenna, Phys. Rev. D 62, 015002 (2000).

[5] N. Arkani-Hamed, S. Dimopoulos, G.F. Giudice, and A. Romanino, Nucl. Phys. B709, 3 (2005).

[6] M. Strassler and K.M. Zurek, Phys. Lett. B 651, 374 (2007).

[7] CDF Note 8701, URL:http://www-cdf.fnal.gov/physics/exotic/r2a/20070208.champ/

[8] DØ Note 4746-CONF, URL:http://www-d0.fnal.gov/Run2Physics/WWW/results/prelim/NP/N27

[9] W. Beenakker, M. Klasen, M. Kramer, T. Plehn, M. Spira, and P. M. Zerwas, Phys. Rev. Lett. 8, 3780 (1999).

[10] A. Arvanitaki, S. Dimopoulos, A. Pierce, S. Rajendran, and J. Wacker, arXiv:hep-ph/0506242.

[11] V.M. Abazov et al. [DØ Collaboration], Phys. Rev. Lett. 99, 131801 (2007). 
[12] V.M. Abazov et al. [DØ Collaboration], Phys. Rev. Lett. 97, 161802 (2006)

[13] T. Adams et al. [NuTeV Collaboration], Phys. Rev. Lett. 87, 041801 (2001).

[14] Paul A.M. Dirac, Proc. R. Soc. A 133, 60 (1931).

[15] A. Abulencia et al. [CDF Collaboration], Phys. Rev. Lett. 96, 201801 (2006). 